\title{
Cyclodextrins in Skin Formulations and Transdermal Delivery
}

\author{
Laszlo Jicsinszky (iD ${ }^{1,}{ }^{*}$ and Giancarlo Cravotto (iD ${ }^{1}$ \\ ${ }^{1}$ Department of Drug Science and Technology, University of Turin, Turin, Italy \\ "Corresponding author: Department of Drug Science and Technology, University of Turin, Turin, Italy. Tel: +39-116707171, Email: ljicsinszky@gmail.com \\ Received 2020 March 11; Revised 2020 April 05; Accepted 2020 April 06.
}

\begin{abstract}
This mini review aims to describe the main physicochemical properties and characteristics of cyclodextrin derivatives (CDs) having been used in various skin-related formulations over the last nearly 50 years. Due to many misunderstandings and inconsistent data on the Internet, the basic properties of CDs, water content, solubility properties, and maximum complexing capabilities are summarized. CDs are grouped by their solubility properties and briefly described by chemical synthesis to reveal the potential contaminants. Soluble and insoluble CD-based polymers are also discussed.
\end{abstract}

Keywords: Solubility, Synthetic Methods, Structure, Cyclodextrin Polymers, Nanosponges

\section{Context}

Cyclodextrins and their derivatives are common drug carriers, and their topical application has gained interest since the beginning of their widespread dissemination in the last decades of the 20th century. Although the application idea of these drug carriers is not new, their physicochemical and major physiological properties are less known for the applicants, scientists, and practitioners outside the specialized laboratories. In this paper, the main chemical characteristics, production methods, and properties of currently used or potentially suitable cyclodextrin derivatives are discussed.

\section{Introduction}

Cyclodextrins (CDs) are natural cyclic oligosaccharides, whose constitution unit is the $\alpha \mathrm{D}$-glucopyranoside. This family of cyclic carbohydrates is often referred to as cyclomaltooligosaccharides, showing the maltose-type glycosidic connection between the glucose units. These constituents are linked together by $\alpha(1 \rightarrow 4)$ glucoside bonds, and although the most commons are the 6,7 , and 8 units containing versions (namely, $\alpha$-, $\beta$-, and $\gamma$ CDs) (1), the largest cyclic structures have more than 150 glucopyranoside units (2).

Recently 3- and 4-membered CDs have also been synthesized (3), but the 5-membered version is the smallest known structure with a cavity (4). These CDs have poor practical importance though they well demonstrate the developments in the oligosaccharide syntheses. While the 6 -10 units containing CDs have truncated cone structures, the hole formed by the carbohydrate units is less distorted in the 6 - 8 membered natural CDs (2). The two hydrophilic hydroxyl rims embrace a relatively less hydrophilic structure, and the cavity is suitable to form adducts with proper chemical structures, like long alkyl chains, aromatic rings, etc.

Owing to the connecting glycosidic oxygens, the formation of H-bonded networks or other non-covalent interactions is possible with appropriate molecules. The two hydroxyl rims are not equally hydrophilic. The secondary hydroxyls form a rigid and strong hydrophilic barrier toward the cavity, while the primary hydroxyls are more flexible, making the rim slightly less hydrophilic. In solution, these primary hydroxyl groups (as $\mathrm{CH}_{2} \mathrm{OH}$ groups) can rotate almost freely, creating a continuously opening and closing door for the cavity. Although their mobility is significantly limited in the solid state, the thermal movement of these groups gives some flexibility to the macrocycle.

The non-covalent associations are generally referred to as "host-guest" or "inclusion" complexes, although in many cases, especially in solutions, the guest molecule has a short average residence time within the cavity. In cases where the guest is large enough, like complex molecules, other macrocycles, oligopeptides, or proteins, the association is restricted to an appropriate part of the large 
molecule (5).

It is also common that the molecular composition is different from the 1:1 host:guest ratio or the complexes create large associations in solutions. The complex stability constant, which characterizes the dynamic equilibrium, is generally within the range of $100-3000 \mathrm{M}^{-1}$, typically around $500 \mathrm{M}^{-1}$ in the case of 1:1 complexes (6). Though this stability constant is apparent because some complex compositions, like 1:1 and 2:2, cannot be distinguished or the low concentrations of other compositions allow their elimination to simplify the calculations.

In many cases, CDs form insoluble complexes, as well. These insoluble complexes have made the ton-scale CD production feasible. The CD production is a green process because not only the raw materials are vegetable polysaccharides, but the waste is also suitable for alcohol production. Isolation techniques of more than 8-membered or the branched CDs, which have one or more glucose-based pendant groups, generally use chromatography, which momentary restricts them into research laboratories.

Cyclofructins are also cyclic oligosaccharides with a cavity, based on fructose, which is also a glucose isomer. Due to their less rigid and symmetric structure, that cavity has somehow different physicochemical properties. However, nowadays, their price has limited their application (7, 8).

Natural ("naked") cyclodextrins have been known for a long time, and numerous CDs have been accepted as drug carriers and included in the GRAS food list for many years (www.fda.gov/food/food-ingredients-packaging/generallyrecognized-safe-gras). The topical toxicity of more common CDs is exhaustively discussed (9). Cyclodextrins have a relatively large margin of safety in all dermal applications and can not only be used exclusively for optimized transdermal drug vehicles for topical or systemic delivery, but also used in skin protection formulations (10) and cosmetics $(11,12)$. CDs generally improve the solubility and/or stability of drugs in topical formulations, promote the transdermal absorption of drugs, and can control the release of active ingredients. However, the penetration of the CDs themselves through the skin is very limited $(7,9$, $13,14)$, which can reduce the drug's side effects (15).

Natural CDs and numerous CD derivatives are chemically stable in a wide range of $\mathrm{pH}$. The lack of reducing end makes the macrocycle extremely stable under basic conditions. It is necessary to note that commercially available native CDs always contain some residual linear dextrins. The reducing-end of these impurities is unstable in the presence of a base and/or higher temperatures, which results in yellow-to-brown coloration. The glycosidic bond can be cleaved easily in strongly acidic solutions $(>0.01 \mathrm{~N}$ concentration of $\mathrm{HCl}, \mathrm{H}_{2} \mathrm{SO}_{4}, \mathrm{HClO}_{4}$, etc.) $(16,17)$, in carboxylic acid solutions at higher temperatures (18), or in anhydrous Lewis acids at moderately high temperatures (19).

Nowadays, many utilizations of CDs can be found on the Internet, starting from their use as catalysts in synthetic chemistry to analytical applications, drug carriers, food ingredients. The general discussion of CD properties, synthesis of their derivatives and their application is well summarized in recent books of Fourmentin et al. $(20,21)$ The idea of application of CDs or their derivatives either in skin formulations (and cosmetics) or as drug carriers for other topical deliveries emerged relatively soon in the blowing era of CD derivatizations (22-26). Application of CDs as solubility enhancers for otherwise poorly soluble small molecules has also become common in the biochemical production processes for a long time (27-29). The first review of CDs in skin applications appeared in the early stages of CD industrialization, but only a few new CDs have been tried since then, and even fewer have become commercialized formulations (23, 24,30-34). Despite their high formulation potential, relatively easy toxicological studies, and early start of publication activity, the skin-related technical papers represent only a minority $(\approx 7 \%)$ in all publications, even within the pharmaceutical and cosmetics subgroup. Due to keywords multiplication in technical papers, the absolute values in Figure 1 are less important.

\section{Discussion}

There are several ways to classify CDs, and due to the limited number of pharmaceutically acceptable CD derivatives, this discussion based on solubility seems reasonable. Since water-soluble CDs are the most used compounds in parenteral and oral formulations, insoluble derivatives can be more suitable for certain extracorporeal formulations, either in pharmaceutical or cosmetic products. Due to the widespread presence of the cosmetics industry, skin products are in a favorable position, and the common interest in the pharmaceutical industry can synergize testing periods.

Table 1 summarizes the most frequently studied CDs, in which solvents are more important from the synthetic view, and the less common organic solvents are ignored. Although even natural CDs are soluble in so-called "dipolar aprotic" organic solvents, their use is less common in the preparation of $\mathrm{CD}$ complexes or formulations. Other 


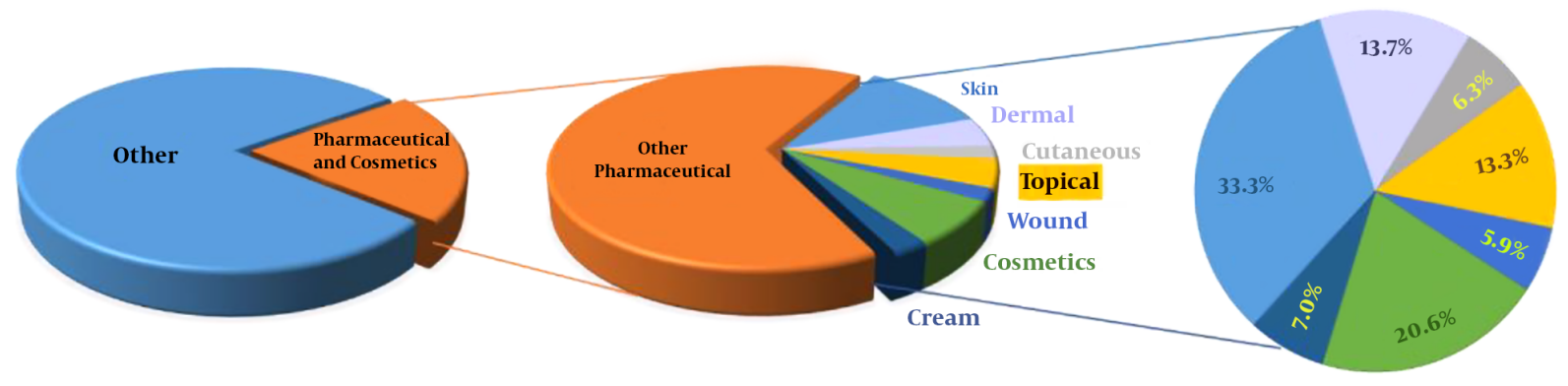

Figure 1. Publication distribution of skin-related formulations by keywords

organic solvents are generally limited to lower alkyl alcohols and acetone.

Usually, the high molecular weight (MW) of naked CDs, as compared to the guest molecules, is a serious drawback. Assuming the most common 1:1 host-guest ratio (which is rarely really achieved in the prepared complexes), the weight ratio of a potential guest molecule is less than $30 \%$ at a theoretical MW of $400 \mathrm{Da}$. Although that ratio is rather more problematic in the parenteral applications than in skin formulations, the MW and dosage of the guest molecule is crucial and restricts the utilization of $\mathrm{CD}$ complexes. Obviously, the derivatization of CDs further reduces this ratio (Table 1), and this is even more important in the cases of polymerized CDs, which can explain the limited number of tested derivatives.

\subsection{Soluble CDs and CD Derivatives}

\subsubsection{Water-Soluble CDs and CD Derivatives}

While natural CDs are crystalline compounds, the most useful randomly derivatized versions are amorphous materials. These CDs are compositions of differently substituted CDs and contain numerous regional isomers, as well. Aqueous solubilities are varied and while the natural $\beta C D$ is relatively poorly soluble $(\sim 2 \%)$ in water, the solubility of randomly decorated variants is almost unlimited. In the shelf-dry form, as available from the suppliers, naked CDs contain a more or less constant amount of water $(10 \%$ - 14\%). Although about half of the crystal water can be readily removed at a moderately high temperature, the complete drying requires more drastic conditions. Most of the water-soluble derivatized CDs contain considerably less residual water, usually in the $3 \%$ - $6 \%$ range, which can likewise be removed under moderate conditions. While the complete drying of both natural and derivatized CDs is relatively easy, water resorption is just as easy.
Although the physicochemical properties of the most common $\alpha$-, $\beta$-, and $\gamma$ CDs are easy to find in various printed and internet sources, many of them are often misunderstood, forgotten or, what is even worse, neglected. The crystal water of natural CDs considerably affects not only the weighing of CDs and aqueous solubilities, but the completely dried CDs are also hygroscopic and very electrostatic materials (36). Additionally, the water-guest molecule exchange is not a one-direction process. Usually, not only the release of the guest molecule needs the active participation of water, but in case of its absence, the formation of the inclusion complex is strongly inhibited, too (37).

A further misunderstanding is the polarity/hydrophobicity of the cavity. Both properties are relative and usually compared to water. "Hydrophobic cavity is suitable to form inclusion complexes with hydrophobic guests" can be often found in the literature and this statement forcibly suggests that only hydrophobic molecules are suitable for the complexation. But this is not the case! Although the first publication on this old scientific problem set the cavity polarity to the n-octanol range, the detailed spectrofluorometric investigation revealed its mid polarity (38-41). The cavity polarity is around the 1:1 (v/v) EtOH-water mixture, which makes this mixture a magic solution which can decompose the majority of complexes.

Hydrophobicity/lipophilicity is another question. This property does not have an absolute scale. Although the most commonly used parameter, $\log \mathrm{P}$ value (logarithm of the partition coefficient $(\mathrm{P})$, between a mixture of water and n-octanol at equilibrium, recently mostly $\log \mathrm{P}_{\mathrm{o} / \mathrm{w}}$, can be experimentally measured, the solubility of the molecules in n-octanol is a serious shortcoming. The $\log \mathrm{P}$ can be computed, and although there are many sophisticated software programs for these calculations, the values 


\begin{tabular}{|c|c|c|c|c|c|c|}
\hline Common Name & $\begin{array}{c}\text { Common } \\
\text { Abbreviation }\end{array}$ & $\begin{array}{c}\text { Average } \\
\text { Substitution }\end{array}$ & $\begin{array}{c}\text { Max. Capacity } \\
{[\mathrm{mmol} / \mathrm{g}]}\end{array}$ & $\begin{array}{l}\text { Approx. Water } \\
\text { Content, } \%\end{array}$ & $\begin{array}{l}\text { Aqueous Solubility, } \\
\text { g/mL water }\end{array}$ & Organic Solvents \\
\hline$\alpha$-cyclodextrin & $\alpha \mathrm{CD}^{\mathrm{e}}$ & & 1.03 & $9-11$ & $\sim 10$ & DMF, DMSO, Py ${ }^{\mathrm{f}}$ \\
\hline$\beta$-cyclodextrin & $\beta C D$ & & 0.88 & $13-14$ & $\sim 2$ & DMF, DMSO, Py \\
\hline$\gamma$-cyclodextrin & $\gamma \mathrm{CD}$ & & 0.77 & $9-11$ & $\sim 15$ & DMF, DMSO, Py \\
\hline $\begin{array}{l}\text { Partially acetylated } \\
\beta C D\end{array}$ & $\mathrm{Ac} 7 \beta \mathrm{CD}$ & $\mathrm{DS}=7-8$ & 0.69 & $3-5$ & $>40$ & $\mathrm{MeOH}, \mathrm{AcOH}$ \\
\hline $\begin{array}{l}\text { Heptakis(2,3,6-tri-o- } \\
\text { acetyl)- } \beta \text { CD }\end{array}$ & $\operatorname{PerAc} \beta C D$ & 21 & 0.50 & $1-2$ & $<1$ & $\begin{array}{c}\mathrm{MeOH}, \mathrm{CHCl}_{3}, \mathrm{CH}_{2} \mathrm{Cl}_{2} \text {, } \\
\text { toluene }\end{array}$ \\
\hline $\begin{array}{l}\text { Crystalline Methylated } \\
\beta \text { CD }\end{array}$ & CRYSMEB & $\mathrm{DS} \approx 4-6$ & 0.83 & $3-5$ & $\sim 10$ & DMF, DMSO, Py \\
\hline Methylated $\beta C D$ & RAMEB $^{g}$ & $\mathrm{DS} \approx 12$ & 0.77 & $2-5$ & $>40$ & DMF, DMSO, $\mathrm{CHCl}_{3}, \mathrm{Py}$ \\
\hline $\begin{array}{l}\text { Heptakis(2,6-di-o- } \\
\text { methyl) } \\
\beta C D\end{array}$ & DIMEB $^{h}$ & $\mathrm{DS}=14$ & 0.75 & $2-5$ & $>30$ & DMF, DMSO, $\mathrm{CHCl}_{3}$, Py \\
\hline $\begin{array}{l}\text { Heptakis(2,6-di-o- } \\
\text { ethyl) } \\
\beta C D\end{array}$ & DIETB $^{i}$ & $\mathrm{DS}=14$ & 0.65 & $<1$ & & DMF, DMSO, $\mathrm{CHCl}_{3}$ \\
\hline $\begin{array}{l}\text { (2-hydroxy) } \\
\text { propylated } \beta \text { CD }\end{array}$ & $\mathrm{HP} \beta \mathrm{CD}$ & $\mathrm{DS} \approx 3-3.5$ & 0.76 & $3-6$ & $>40$ & $\mathrm{MeOH}, \mathrm{EtOH}$ (limited) \\
\hline $\begin{array}{l}\text { (2-hydroxy) } \\
\text { propylated } \beta \mathrm{CD}\end{array}$ & $\mathrm{HP} \beta \mathrm{CD}$ & $\mathrm{DS} \approx 4-5$ & 0.72 & $3-6$ & $>40$ & $\mathrm{MeOH}, \mathrm{EtOH}$ \\
\hline $\begin{array}{l}\text { (2-hydroxy) } \\
\text { propylated } \beta C D\end{array}$ & $\mathrm{HP} \beta \mathrm{CD}$ & $\mathrm{DS} \approx 6-7$ & 0.66 & $3-6$ & $>40$ & $\mathrm{MeOH}, \mathrm{EtOH}$, acetone \\
\hline $\begin{array}{l}\text { (2-hydroxy) } \\
\text { propylated } \gamma \text { CD }\end{array}$ & $\mathrm{HP} \gamma \mathrm{CD}$ & $\mathrm{DS} \approx 4-5$ & 0.64 & $3-6$ & $>40$ & MeOH, EtOH (limited) \\
\hline $\begin{array}{l}\text { (2-hydroxy) ethylated } \\
\beta C D\end{array}$ & $\mathrm{HE} \beta \mathrm{CD}$ & $\mathrm{DS} \approx 4-5$ & 0.75 & & $>40$ & $\mathrm{MeOH}$ \\
\hline $\begin{array}{l}\text { Carboxymethylated } \\
\beta C D \text { sodium salt }\end{array}$ & $\mathrm{CM} \beta \mathrm{CD}^{\mathrm{j}}$ & $\mathrm{DS} \approx 3.5-4.5$ & 0.69 & $3-6$ & $>30$ & $\mathrm{MeOH}$ (limited) \\
\hline $\begin{array}{l}\text { Carboxymethyl- } \\
\text { ethylated } \beta \text { CD sodium } \\
\text { salt }\end{array}$ & $\mathrm{CME} \beta \mathrm{CD}^{\mathrm{j}}$ & $\mathrm{N} / \mathrm{A}$ & $\mathrm{N} / \mathrm{A}$ & $\mathrm{N} / \mathrm{A}$ & $\mathrm{N} / \mathrm{A}$ & \\
\hline $\begin{array}{l}\text { Carboxyethylated } \beta \mathrm{CD} \\
\text { sodium salt }\end{array}$ & $\mathrm{Ce} \beta \mathrm{CD}^{\mathrm{j}}$ & $\mathrm{DS} \approx 3.5-4.5$ & 0.66 & $3-6$ & $>30$ & \\
\hline $\begin{array}{l}\text { Sulfobutylated } \beta C D \\
\text { sodium salt }\end{array}$ & $\mathrm{SBE} \beta \mathrm{CD}^{\mathrm{j}, \mathrm{k}}$ & $\mathrm{DS} \approx 6-7$ & 0.51 & $3-6$ & $>30$ & \\
\hline Soluble $\beta$ CD polymer ${ }^{1}$ & $\beta$ CDPS & $\mathrm{N} / \mathrm{A}$ & 0.58 & $3-6$ & $>30$ & DMF, DMSO \\
\hline $\begin{array}{l}\text { Ionic soluble } \beta \text { CD } \\
\text { polymer }^{1}\end{array}$ & $\begin{array}{c}\beta \text { CDPSI } \\
\text { CM } \beta \text { CDPSI }\end{array}$ & $\mathrm{N} / \mathrm{A}$ & 0.58 & $3-6$ & $>30$ & DMF, DMSO \\
\hline $\begin{array}{l}\text { Insoluble } \beta \mathrm{CD} \\
\text { polymer }^{1}\end{array}$ & $\beta$ CDPI & $\mathrm{N} / \mathrm{A}$ & 0.58 & $1-2$ & $\mathrm{~N} / \mathrm{A}$ & \\
\hline Nanosponge $^{m}$ & NS & $\mathrm{N} / \mathrm{A}$ & ND & ND & $>30$ & DMF, DMSO \\
\hline
\end{tabular}

Abbreviations: $\mathrm{CH}_{2} \mathrm{Cl}_{2}$ : methylene chloride; $\mathrm{CHCl}_{3}$ : chloroform; DMSO: dimethyl sulfoxide; DMF: N,N-dimethyl formamide; EtOH: ethanol; MeOH: methanol; N/A: not applicable; ND: no data; Py: pyridine; SBE: sulfobutyl ether.

${ }^{\mathrm{a}}$ The randomly (statistically) derivatized CDs are characterized by DS (degree of substitution = average number of substituents/ring, pharmacopeia recommended cal-

culation from 1H-NMR). Due to quality assurance reasons the producers often give a wide range for DS (sometimes as substituent/glucose unit!).

${ }^{\mathrm{b}}$ The middle values of DS are used, polymers are calculated with $60 \%$ CD content.

${ }^{\mathrm{c}}$ From product catalogs of main CD suppliers; the exact value is the certificate of analysis.

${ }^{\mathrm{d}}$ Shelf-dry, as can be received from suppliers.

${ }^{\mathrm{e}}$ Sometimes CyD is also used.

${ }^{\mathrm{f}}$ Poorly soluble complex formation with the solvent.

${ }^{g}$ Rarely $\mathrm{M} \beta \mathrm{CD}, \mathrm{PM} \beta \mathrm{CD}$, or $\mathrm{RM} \beta \mathrm{CD}$ is used, as of methyl-, partially methylated, or randomly methylated $\beta \mathrm{CD}$.

${ }^{\mathrm{h}}$ Many differently methylated derivatives use this name. The most common product is the DIMEB50, which contains $<40 \%$ heptakis(2,6-di-O-methyl)- $\beta$ CD, despite its unreasonably low heptakis content. The larger number after "DIMEB" refers to higher heptakis content, e.g. DIMEB95 contains > 95\% heptakis(2,6-di-O-methyl)- $\beta$ CD.

${ }^{\mathrm{i}}$ Abnormal solubility-temperature profile. Solubility at $100^{\circ} \mathrm{C}<2 \%$.

${ }^{\mathrm{j}}$ As the sodium salt.

${ }^{\mathrm{k}}$ Sometimes $\mathrm{SB} \beta \mathrm{CD}$ is also used, because "E" is useless and redundant for "ether" and missing in the nicknames of HE/ET/HP/ME/CM, etc. derivatives.

${ }^{1}$ Epichlorohydrin crosslinked CD polymer, CD content is around $55 \%-60 \%$.

${ }^{\mathrm{m}}$ Various derivatives; many different kinds of linkers are in this class, see the text and the Trotta's review (35). 
of different programs do not always match. Experimental data on various complexes provide good evidence for the discrepancy between the declared hydrophobic cavity and the complexation of water (37), inorganic $(42,43)$ and organic, particularly sulfonic acid, salts (39). As a conclusion: the CD cavity is only considerably less hydrophilic than the hydroxyl rims of CDs or the aqueous macro-environment.

The high hydrophilicity of water-soluble CDs, as a big advantage, limits their absorption through the skin. It is necessary to mention that despite the unchanged number of hydroxyl groups, the (2-hydroxy)propyl derivatives are less hydrophilic than the parent CDs. The methyl substitution, despite the lipophilic methyl groups, increases the aqueous solubility. The explanation is the modification of the rigid hydrophilic secondary $\mathrm{OH}$-rim: methylation reduces the ability to form hydrogen-bridged macromolecular associates between CDs. Water-soluble CDs are also suffering a noticeable disadvantage: the solubility in water. Without protection, like in patches, CDs and their complexes are readily removed from the skin, reducing the effective absorption of the active ingredient.

However, the wash-off phenomenon is a beneficial effect: the accumulation of CDs on the body surface is prevented. In the mid-1990s, there was a brief, but rather a self-evident, debate about the accumulation of carcinogens in the gastrointestinal tract by CDs of oral drug formulations $(44,45)$. Considering the basic physicochemical processes, it was more about money than a real threat. The complex formation is a concentration-driven dynamic equilibrium. It is difficult to believe that the picomolar, but rather less, presence of harmful chemicals can considerably be complexed, and so they could be accumulated by CDs. Although the accumulation of undesirable substances on the skin may be more pronounced, it is less likely than in the mentioned case. Additionally, exactly water solubility of CDs is what can eliminate this threat, and they are washed off before the accumulation of any components from the environment.

How are CDs prepared? Although the pure chemical synthesis of CDs is solved, that way is nothing else than an interesting challenge for organic synthetic chemists. The preparation of CDs is still based on a more than 100 years methodology (1), only the living organism is substituted with isolated, sometimes genetically engineered and fine-tuned, enzymes (46). The cyclization of the preformed amylose fragments is done by various enzymes, but dominantly a variant of cyclodextrin glucosyltransferase (CGTase). This type of enzymes are also known as cyclodextrin glycosyltransferase or cyclodextrin glucan- otransferase, but because CDs are constructed per definitionem from $\alpha(1 \rightarrow 4)$ linked glucosides, the first alternative is more correct.

The book of Jin (47) is a basic source on the enzymatic production of CDs. The isolation of products depends on the use or lack of organic complexants or solvents in the conversion mixture. The traditional synthesis allows the preparation of isotope labeling of CDs in the "core". In isolated environments, the enhancement of ${ }^{14} \mathrm{C}$ isotope as $\mathrm{CO}_{2}$ is built into the constituting glucoside units. Of course, the lack of control means that the incorporation of the isotope is necessarily random (48). The complete elimination of the complexant from the products from the conversion mixture is crucial (49). The incomplete removal of toxic organics has led to the stigmatization of CDs as toxic materials by the first misinformation on the toxicity of CDs (50). Although it is true that not all CDs, like not all inorganic or organic materials, can be administered parenterally, but CDs themselves are not ab ovo toxic substances, as can be seen in various medical, food, cosmetics, or other household utilizations.

Apart from naked CDs, among the studied CD derivatives the ionic and soluble polymeric derivatives are soluble almost exclusively in water. These are the carboxymethylated (CM), carboxyethylated (CE), sulfobutylated (SB), and polymerized CDs. It is also true that nanosponges may contain some acidic end groups and those derivatives are considered neutral. Although most of the water-soluble CDs are also soluble in some organic solvents, in this section, the DMF, DMSO, or pyridine-like solvents are excluded. The removal of those chemicals after derivatization reactions, or exclusion from formulations, is an obvious demand.

Ionic $\mathrm{CD}$ derivatives are almost insoluble in all organic solvents either in ionized or protonated forms, although counterions can affect their solubility. The ionic forms of both carboxylic and sulfoalkylated CDs are chemically stable. They are very hydrophilic molecules, and the dominant substitution location is on the secondary hydroxyl side. The protonated form makes these derivatives chemically labile. Organic acid (carboxylic acid) derivatives are capable of reversibly forming molecular and molecular esters, and sulfonic acids are strong acids and can destroy the macrocycle. The general synthetic procedure of neutral and ionic alkylated CDs is summarized in Figure $2 \mathrm{~A}$.

Soluble CD polymers (CDPS) have complicated 3D structures. Not only because of the different reactivities of the CD hydroxyls, but the bifunctionality of the linkers inheritably results in sidechains that cannot be attached 
A

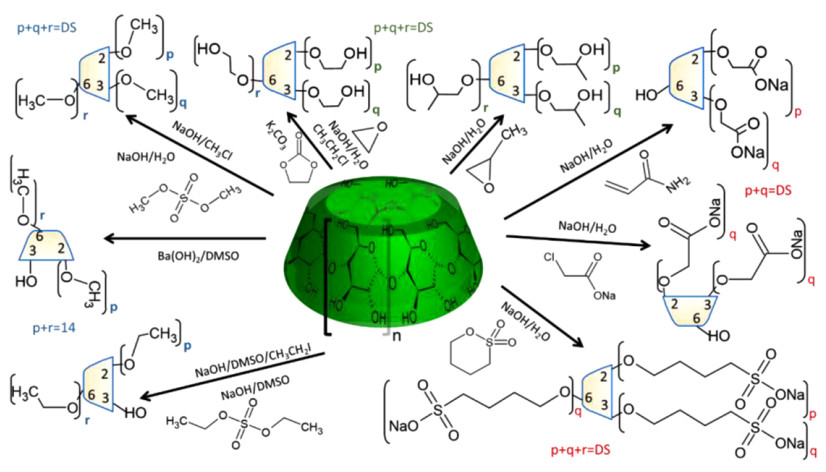

B

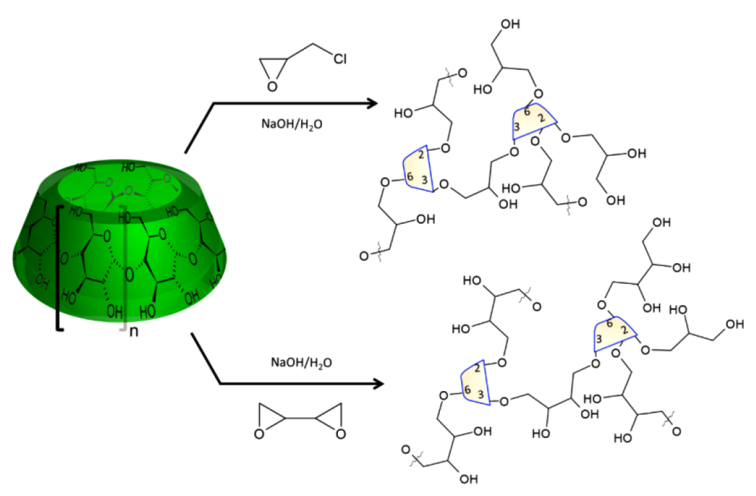

C
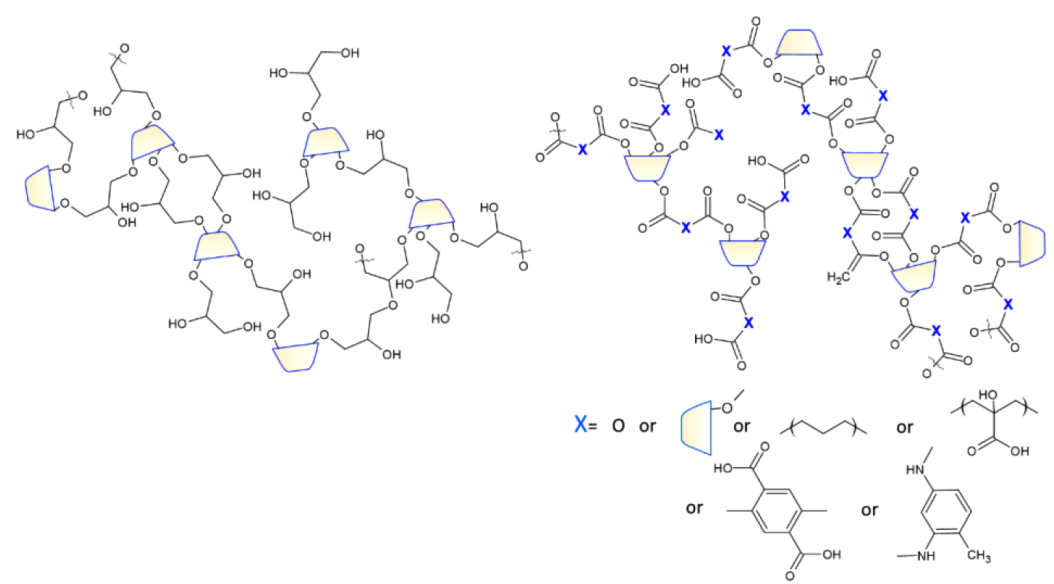

CD Polymer

CD Nanosponge

Figure 2. General synthetic method of the water-soluble A, monomeric; B, polymeric cyclodextrin derivatives; C, the major structural differences between CDPS and nanosponges. $\mathrm{N}=0 \alpha \mathrm{CD} ; \mathrm{n}=1 \beta \mathrm{CD} ; \mathrm{n}=2 \gamma \mathrm{CD}$.

to other CDs. Although "linear" CD polymers have also been synthesized (51), the complex synthesis shifts their use in potential formulations to the distant future, if possible at all. In general, polymerized CDs can be divided into two major subgroups: $\mathrm{CD}$ polymers (CDP) meaning that usually the chemically stable bond crosslinks CDs (52, 53), and the hydrolyzable nanosponges (NS) (35). The synthetic routes of ether-linked CDPs are shown in Figure 2B, while the syntheses of dominantly acyl group-linked NS are in Figure 3. There is a third, momentarily very minor 
group but in chemical terms in the neighborhood of NS: the co-polyesters of multicarboxylic acids, like citric, glutaric, adipic, etc. acids $(35,54,55)$.

A cuckoo nest inside the NS is the hemiacetal-linked version that is much more labile than esters and is degraded even by very weak acids, too. Despite their similarity to the NS, their application is very limited (55). The acidic crosslinkers form esters, but the acidic end groups can also trigger their acid-catalyzed hydrolysis, which can considerably modify the polymeric structure. Due to the poorly characterized structures and the few applications, particularly in the skin and cosmetics area, their synthetic method is mentioned tangentially only in Figure 3. The poor reproducibility of the synthesis and aging are the most common problems of CDPs. The reactive end groups of the bifunctional chemical reagents react not only with CDs but also with water, even in the solid products. Because of the poor chemical characterization, the substitution patterns in these CDs are not clear.

Some noncovalent associations, like rotaxanes, are also called CD-polymers (56). Even though the complexed component is polymeric, these $\mathrm{CD}$ derivatives are not $\mathrm{CD}$ polymers. In some cases, the polymeric backbone attached to CDs are also classified as CD polymers, but they are rather polymers with some $\mathrm{CD}$ content than $\mathrm{CD}$ polymers.

Through the combination of the polymerization reaction with another alkylation, like methylation or carboxyalkylation, the microenvironment can be readily finetuned, at least theoretically. Komiyama (57), the contemporary "father" of modern CD chemistry, presented the outlines of molecular imprinting, which was recently reviewed by Lay et al. (58). The linkers are dominantly located on the secondary hydroxyl rims, and often CD units bear many (2,3-dihydroxy)propyl or, in general, $(2, \omega, \omega$ trihydroxy)alkyl sidechains. The synthetic route of CDPs is shown in Figure 2B.

NSs are prepared by the reaction of CDs with various activated carbonyl compounds, like triphosgene, dicarbonates, carbonyldiimidazole, dianhydrides, diisocyanates, or 2,2-bisacrylamidoalkyl acids. These reactions result in carboxylic derivatives, and the syntheses show similarities to the amphiphilic CD esters, as seen in Figure 3. In NS typically three kinds of chemical bonds connect CDs and linkers: ester (35), carbamate (59), and eventually amide (60). The latter is not classical NS, because the ether-bound linkers are connected to each other with amide bonds.

Although numerous publications are discussing the application of NS, their spread is still poor despite their sometimes excellent physicochemical properties. The lim- ited number of applications are not exclusively bound to their poor shelf-life or weak synthetic reproducibility, but rather the preparation of numerous different derivatives without a lead. The review of Tejashri et al. (54) well summarizes the variability of NS among the potential drug formulations. The numerous CDs in NS increase the MW, which further worsens the host:guest mass ratio. Although it is difficult to find general considerations for the substitution patterns in various NSs, the preparation methods suggest similarities to the alkyl-type soluble polymers. The general structural differences between $\mathrm{CD}$ polymers and NS, without the (hemi)acetal version, are shown in Figure $2 \mathrm{C}$.

Although the poor guest weight ratios in CDP formulations are less suitable for parenteral, oral, or mucosal delivery, it is less important in dermal applications (61) or for the less price-sensitive cosmetics.

\subsubsection{Derivatives Soluble in Water and Organic Solvents}

These amphiphilic CD derivatives are suitable for both solubilization of drugs and their controlled release. Their aqueous solubility allows their easy removal from the skin, which is especially useful in cosmetics and skin care products. The good solubilization properties also decrease the possible overdosing. The associations between the CD molecules are greatly reduced by the breaking of the symmetry and hydrogen bonds in the rigid hydrophilic rim, which thereby provides an increased aqueous solubility and a larger flexible cavity. Some derivatives are soluble not only in alcohols but also in acetone and toluene, which is useful in their purification processes.

It is necessary to mention that CDs in this section are not "amphiphilic" CD derivatives. "Amphiphilic" CDs form a subgroup of differently substituted CDs, containing both hydrophilic (hydroxyalkyl/carboxyalkyl) and hydrophobic (fatty acid esters, -carbamates) moieties. Although those CD derivatives are tested in various targeted drug delivery formulations, their appearance in dermal formulations is negligible.

Only a few derivatives are in this class: the (2hydroxy)propylated, the partially acetylated, and the $>13$ methyl groups substituted CDs (actually, only the DIMEB). The increasing DS moves the HP-CD solubility from $\mathrm{MeOH}$ toward the longer alkyl alcohols. While the oldest DS $\approx 3$ - 3.5 HP $\beta \mathrm{CD}$ is freely soluble in $\mathrm{MeOH}$ and partially only in $\mathrm{EtOH}$, the $\mathrm{DS} \approx 4.5$ is well soluble in $\mathrm{MeOH}$ and $\mathrm{EtOH}$, and the $\mathrm{DS} \approx 6.5$ is freely soluble in EtOH. A similarly increased solubility can be found in acetone, which is widely used to remove the formed 1,2-propylene glycols in their produc- 


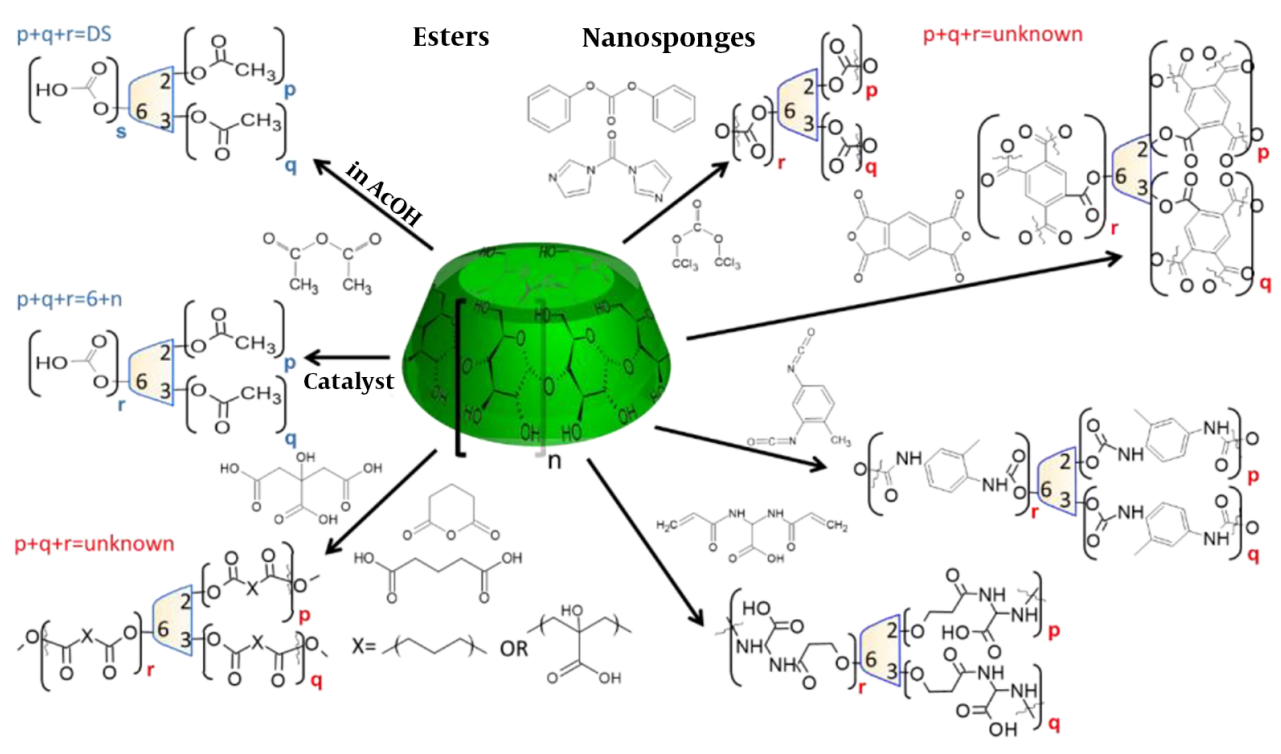

Figure 3. General synthetic method of acylated $\mathrm{CD}$ derivatives and nanosponges. $\mathrm{N}=0 \alpha \mathrm{CD} ; \mathrm{n}=1 \beta \mathrm{CD} ; \mathrm{n}=2 \gamma \mathrm{CD}$

tion.

Since the low DS HPP $\beta$ CDs can be "recrystallized" from $\mathrm{MeOH}$ by the addition of acetone, the $\mathrm{DS} \approx 6.5 \mathrm{HP} \beta \mathrm{CD}$ is freely soluble in acetone. Of course, in aqueous solutions of these solvents, those derivatives have unlimited solubility. Partially acetylated CDs are freely soluble in water and $\mathrm{MeOH}$, and even also in toluene, but the insoluble toluene complex starts to precipitate within a short period. This complexation can be utilized in purification processes. The synthesis of the partially acetylated CD derivatives is shown in Figure 3. Although DIMEB is well soluble in $\mathrm{MeOH}$ (and acetone) until the heptakis(2,6-di-O-methyl) $\beta$ CD content is low ( $<55 \%-60 \%$ ), the increasing heptakis content considerably decreases its solubility. The solubility of DIMEB in acetone is tricky: after dissolution, as the poorly soluble acetone complexes formed, the heptakis- and \pm 2 methylated CDs begin to precipitate soon. Recrystallization of the DIMEB/acetone complex from $\mathrm{MeOH}$ increases the heptakis content up to $85 \%-90 \%$ and removes the acetone. However, RAMEB, which contains only $<13$ methyl groups on the macrocycle, is poorly soluble in anhydrous alcohols and acetone. Methylated CDs from DS $\approx 8$ are also soluble in halogenated organic solvents, which can be utilized in their purification processes.

\subsection{Derivatives Are Soluble in Organic Solvents Only}

The number of organic solvent-soluble CD derivatives reported in skin applications has been restricted for a long time to the heptakis(2,6-di-O-ethylated) (DIETB) (62$64)$ and the peracetylated (65) $\beta$ CDs (PerAcCDs) only. The syntheses of these CDs are summarized in Figures $2 \mathrm{~A}$ and 3. These CDs are insoluble in water, and the hydrophilic profile of the naked CDs is reversed: the cavity is more hydrophilic than the substituted hydroxyl rims. The relative higher hydrophilicity of the cavity allows the controlled release of hydrophilic drugs, although it is also true that their penetration through the skin is very limited $(14,62$ 65). These CDs are appropriate for topical applications, which are mainly targeted by the cosmetic and skin care formulations.

Dried PerAcCDs are soluble in water-free alcohols, acetone, halogenated solvents, and even in toluene. Since PerAcCDs are capable of forming a complex with water, this complex formation significantly reduces their alcohol solubilities and accelerates the formation of the toluene complex (37). The toluene complexation is depending on the residual water content, which allows for the effective removal of the synthesis impurities. The preparation of DIETB requires more sophisticated reaction conditions and complicated work-up processes, using different organic solvents than the PerAcCDs. The preparation is similar to the methylated version, as seen in Figure 2A. The highly hydrophobic DIETB makes its purification difficult, which causes limited application potential. Since the crude product is soluble in hexanes, the heptakis version slowly precipitates, leaving the residual reagent, solvents, and the 
majority of the randomly substituted versions dissolved. Although this results in a higher heptakis content as compared to DIMEB, further purification is more difficult and increases its production costs.

\subsection{Insoluble CD Derivatives}

The insoluble CD polymers (CDPIS) have a 3D network of CDs linked by the spacer and are practically insoluble in all solvents. The insoluble nature of these $\mathrm{CD}$ derivatives also restricts their non-destructive analytical studies, which turns their structural characterization to a complicated task. Although the synthesis of CDPIS uses the same linker as the soluble ones, the number of linkers, their spatial arrangement, and the surface components are even more difficult to determine. The synthetic methods are practically identical with the soluble versions shown in Figure $2 \mathrm{~B}$, only the $\mathrm{CD} /$ crosslinker molar ratio is different. The production of well-defined structures with uniform particles is usually very complicated, though this complexity is rather technical than chemical. Surfactants, such as alkanols (e.g., n-butanol) and/or mineral oils are applied to control the particle sizes of the product in the polymerization reaction, resulting in nice, uniform beads, like the ion-exchanger resins, as in Figure 4A (53, 66-68). Although these beads have excellent adsorption properties, they are physically quite vulnerable. Intensive physical destruction of the beads occurs upon dehydration, caused by either solvents or moderately high temperature. The preparation method of the insoluble bead polymer is not obvious, and the hard reproducibility of the synthesis results in difficult accessibility from commercial sources. Although the bead polymer is useful in various preparations, its usability in skin formulations is limited.

The powder form of CDPIS is more suitable for various skin formulations. Under the conventional reaction conditions, the powder is complicated to prepare. Recently, a solvent-free, green chemical method (69) has been published for the production of standardized CDPIS powder, which is likely to provide greater potential for CDPIS in extracorporeal preparations. The powdered form is prepared more effectively with a reduced $\mathrm{CD} /$ reagent ratio, relative to the bead polymer. Although CDPIS powder has a slightly lower complexing ability than the pearls, due to its advantageous physicochemical properties, it can range from medical applications to skin care and cosmetics by the advantage of controlled weighing and uniformity. Small particles (Figure 4B) are also suitable for the controlled release of drugs. Particles have almost zero zeta-potential in water that can be finely tuned with electrolytes to reach favorable association properties of the powder.

Insoluble ester-type nanosponge, citric acid linked $\beta \mathrm{CDs}$, has also been reported (70). Although the solid nanosponge chemical stability is better than the soluble ones, the hydrolyzable chemical bond restricts a wider utilization. The characterization of insoluble nanosponges is even less sophisticated than the soluble versions.

\section{Conclusions}

The poor transdermal absorption of CDs makes them a promising drug ingredient in skin preparations. Over the past nearly 50 years, the number of $C D$ derivatives in dermal compositions has remained essentially unchanged. This is somehow understandable by the small number of pharmaceutically acceptable CDs, although a larger number of newly targeted CD derivatives could have been expected due to the milder toxicological requirements. Many CDs are widely used in everyday products, and their proliferation in skin products will also be evident soon. The resolution of the difficult, sometimes problematic, laboratorylevel synthetic reproducibility and full characterization of CDPIS could be a big step in dermal utilizations, but this requires further intensive research.

\section{Acknowledgments}

The University of Turin is warmly acknowledged for its financial support (Fondi Ricerca Locale 2019 and 2020).

\section{Footnotes}

Authors' Contribution: Study of concept, literature collection and compilation LJ and GC.

Conflict of Interests: The authors declare no conflict of interest.

Funding/Support: University of Turin, Turin, Italy funded this research (Fondi Ricerca Locale 2019 and 2020). 

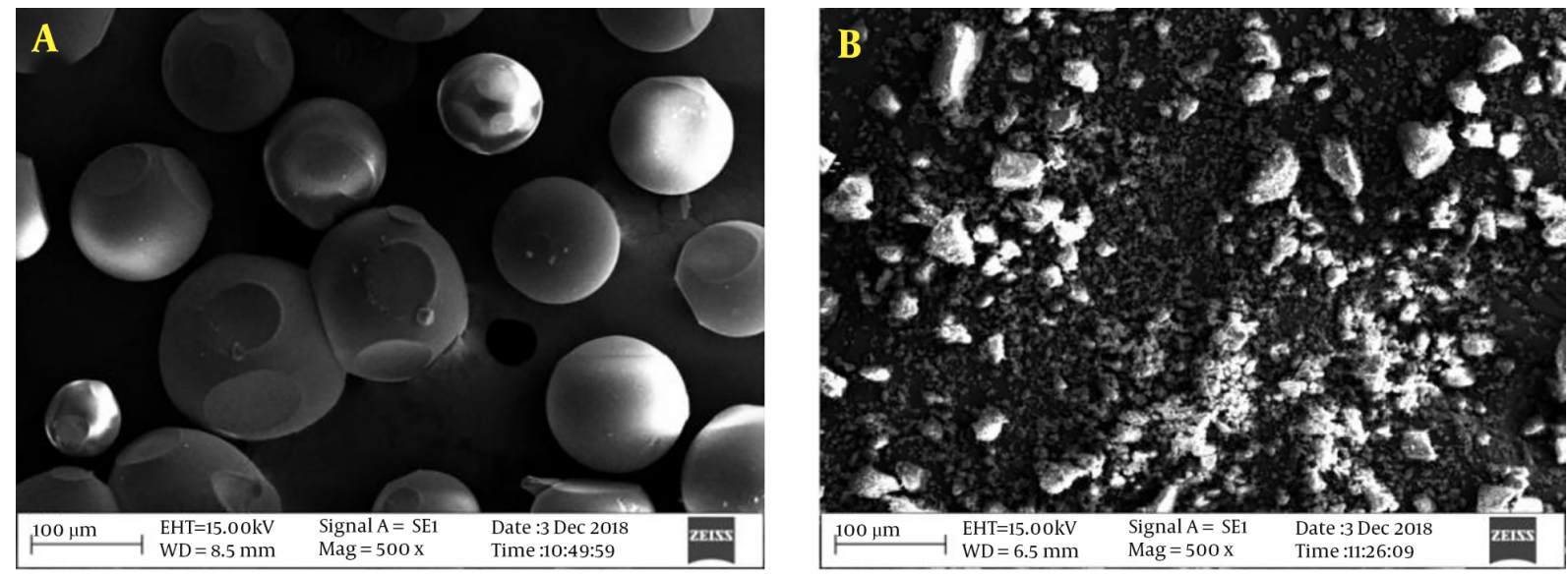

Figure 4. A, SEM pictures of the bead; and B, the powder CDP.

\section{References}

1. Szejtli J, Osa T, Atwood JL. Cyclodextrins. In: Atwood JL, Davies JED, MacNicol DD, Vögtle F, editors. Comprehensive supramolecular chemistry. 3. Ixfoord: Elsevier; 1996.

2. Jicsinszky L. Large ring cyclodextrins. Cyclodextrin N. 2005;19(11):27982.

3. Ikuta D, Hirata Y, Wakamori S, Shimada H, Tomabechi Y, Kawasaki Y, et al. Conformationally supple glucose monomers enable synthesis of the smallest cyclodextrins. Science. 2019;364(6441):674-7. doi: 10.1126/science.aaw3053. [PubMed:30975766].

4. Nakagawa T, Ueno K, Kashiwa M, Watanabe J. The stereoselective synthesis of cyclomaltopentaose. A novel cyclodextrin homologue with D.P. five. Tetrahedron Letters. 1994;35(12):1921-4. doi: 10.1016/s00404039(00)73196-0.

5. Moses LR, Dileep KJ, Sharma CP. Beta cyclodextrin-insulinencapsulated chitosan/alginate matrix: Oral delivery system. J Appl Polymer Sci. 2000;75(9):1089-96. doi: 10.1002/(sici)10974628(20000228)75:9<1089::aid-app1>3.0.co;2-5.

6. Connors KA. Population characteristics of cyclodextrin complex stabilities in aqueous solution. J Pharm Sci. 1995;84(7):843-8. doi: 10.1002/jps.2600840712. [PubMed: 7562435].

7. Immel S, Schmitt GE, Lichtenthaler FW. Cyclofructins with six to ten $\beta$-( $1 \rightarrow 2)$-linked fructofuranose units: Geometries, electrostatic profiles, lipophilicity patterns, and potential for inclusion complexation1 Molecular modeling of saccharides, Part 19. For Part 18, see ref.[1].1. Carbohydr Res. 1998;313(2):91-105. doi: 10.1016/s00086215(98)00208-0.

8. Chen Y, Yang L, Feng F, Ge Q, Guo D, Chen Y. Pharmaceutical compositions. Google Patents; 2016. Report No.: EP2758052A2.

9. Cal K, Centkowska K. Use of cyclodextrins in topical formulations: Practical aspects. Eur J Pharm Biopharm. 2008;68(3):467-78. doi: 10.1016/j.ejpb.2007.08.002. [PubMed:17826046].

10. Fenyvesi E, Jicsinszky L, Szejtli J, Schwarzenbach R. Inclusion complexes of UV Filters in solution and in solid state. In: Labandeira J, VilaJato J, editors. 9th Proc Int Symp Cyclodextrins. Berlin, Germany: Kluwer Academic Publishers; 1999. p. 639-43. doi: 10.1007/978-94-011-4681-4_151.

11. Blasi P, Schoubben A, Giovagnoli S, Rossi C, Ricci M. The real value of novel particulate carriers for sunscreen formulation. Expert Rev Der- matol. 2014;6(5):509-17. doi: 10.1586/edm.11.57.

12. Allegre M, Deratani A. Cyclodextrin uses: From concept to industrial reality. Agro Food Ind Hi-Tech. 1994;5:9-17.

13. Ogiso T, Paku T, Iwaki M, Tanino T, Nishioka S. Percutaneous ab sorption of physiologically active peptides, ebiratide and elcatonin in rats. Biol Pharm Bull. 1994;17(8):1094-100. doi: 10.1248/bpb.17.1094. [PubMed: 7820115]

14. Williams AC, Shatri SR, Barry BW. Transdermal permeation modulation by cyclodextrins: A mechanistic study. Pharm Dev Technol. 1998;3(3):283-96. doi: 10.3109/10837459809009856. [PubMed: 9742549].

15. Uekama K, Hirayama F, Irie T. Cyclodextrin drug carrier systems. Chem Rev.1998;98(5):2045-76. doi:10.1021/cr970025p. [PubMed: 11848959].

16. Szeman J, Szente L, Szabo T, Jicsinszky M, Vikmon L. ß-cyclodextrin methyl ethers, structure/activity relationship. In: Hedges A, editor Minutes of 6th Int Symp Cyclodextrins. Paris: Éditions de Santé; 1992. p. 345-50.

17. Szejtli J, Budai Z. Acid hydrolysis of $\beta$-cyclodextrin. Acta Chim Acad Sci Hung. 1976;91:73-80.

18. Griskonis E, Vaitkus R. Investigation of some thermodynamic parameters of acidic hydrolysis $\beta$-cyclodextrin in the presence of inclusion complexes. Org React.1997;31:27-30.

19. Jicsinszky L, Szejtli J. Effect of catalysts on the acylation of cyclodextrins. In: Hedges AR, editor. Minutes of 6th Int Symp Cyclodextrins. Paris: Éditions de Santé;1992. p. 96-100. doi:10.13140/rg.2.2.26212.78729.

20. Fourmentin S, Crini G, Lichtfouse E. Fourmentin S, Crini G, Lichtfouse E, editors. Cyclodextrin fundamentals, reactivity and analysis. Germany: Springer; 2018. doi: 10.1007/978-3-319-76159-6.

21. Fourmentin S, Crini G, Lichtfouse E. Cyclodextrin applications in medicine, food, environment and liquid crystals. Environmental chemistry for a sustainable world. 17. Germany: Springer; 2018

22. Másson M, Pitha J, Loftsson T. Synthesis of cyclic glycerol ether cyclodextrin derivatives and investigation of their binding properties with drugs. J Incl Phenom Macrocyclic Chem. 1999;33(4):459-67. doi: 10.1023/a:1008050825635.

23. Duchene D, Wouessidjewe D, Poelman MC. Cyclodextrins in cosmetics. Cosmet Sci Technol Ser. 1999;19:275-93.

24. Duchene D, Wouessidjewe D, Poelman MC. Cyclodextrins for dermal dosage forms. In: Seiller MC, Martini M, editors. . Formes Pharm Appl Locale; 1996. p. 481-99. 
25. Duchêne D, Wouessidjewe D, Poelman MC. Duchêne D, editor. New trends in cyclodextrins and derivatives. Paris: Editions de Santé;1991.

26. Szeman J, Ueda H, Szejtli J, Fenyvesi E, Watanabe Y, Machida Y, et al. Enhanced percutaneous absorption of homogenized tolnaftate/betacyclodextrin polymer ground mixture. Drug Des Deliv. 1987;1(4):32532. [PubMed: 3509341].

27. Szejtli J. Cyclodextrins in biotechnology. Starch Stärke.1986;38(11):38890. doi: 10.1002/star.19860381107.

28. Kalia VC, Patel SKS, Kang YC, Lee JK. Quorum sensing inhibitors as antipathogens: Biotechnological applications. Biotechnol Adv. 2019;37(1):68-90. doi: 10.1016/j.biotechadv.2018.11.006. [PubMed: 30471318].

29. Seidel GJ. Modifying oocytes and embryos to improve their cryopreservation. Theriogenology. 2006;65(1):228-35. doi: 10.1016/j.theriogenology.2005.09.025. [PubMed: 16263160].

30. Gidwani B, Vyas A. Synthesis, characterization and application of epichlorohydrin-beta-cyclodextrin polymer. Colloids Surf B Biointerfaces. 2014;114:130-7. doi: 10.1016/j.colsurfb.2013.09.035. [PubMed: 24185192].

31. Haimhoffer A, Rusznyák A, Réti-Nagy K, Vasvari G, Váradi J, Vecsernyés $M$, et al. Cyclodextrins in drug delivery systems and their effects on biological barriers. Sci Pharm. 2019;87(4):33. doi: 10.3390/scipharm87040033.

32. Mohapatra S, Sapra O, Paroha S, har. Impact of cyclodextrin in drug delivery system. Sust Agric Rev. 2018;27:271-93. doi: 10.1007/978-3-31975190-0_10.

33. Loftsson T, Masson M. Cyclodextrins in topical drug formulations: Theory and practice. Int I Pharm. 2001;225(1-2):15-30. doi: 10.1016/s0378-5173(01)00761-x. [PubMed: 11489551].

34. Muankaew C, Loftsson T. Cyclodextrin-based formulations: A noninvasive platform for targeted drug delivery. Basic Clin Pharmacol Toxicol. 2018;122(1):46-55. doi: 10.1111/bcpt.12917. [PubMed: 29024354].

35. Trotta F, Zanetti M, Cavalli R. Cyclodextrin-based nanosponges as drug carriers. Beilstein J Org Chem. 2012;8:2091-9. doi: 10.3762/bjoc.8.235. [PubMed: 23243470]. [PubMed Central: PMC3520565].

36. Jicsinszky L. Some comments on the cyclodextrin solubilities. MOJ Bioorg Org Chem. 2019;3:11-3. doi:10.15406/mojboc.2019.03.00091.

37. Jicsinszky L, Martina K, Caporaso M, Cintas P, Zanichelli A, Cravotto G. Complexes of peracetylated cyclodextrin in a non-aqueous aprotic medium: the role of residual water. Phys Chem Chem Phys. 2015;17(26):17380-90. doi: 10.1039/c5cp02379c. [PubMed: 26077122].

38. Street KW. Cyclodextrin cavity polarity and chromatographic implications. J Liq Chromatogr. 1987;10(4):655-62. doi: 10.1080/01483918708069016.

39. Chun IK. Quantitative estimation of binding site polarities of watersoluble polyparacyclophanes in aqueous solution by spectrofluorometry. J Pharm Invest. 1991;21(3):133-41.

40. Biczok L, Jicsinszky L, Linschitz $\mathrm{H}$. Solvent-dependent radiationless transitions in fluorenone: A probe for hydrogen bonding interactions in the cyclodextrin cavity. J Inclusion Phenom Mol Recognit Chem. 1994;18(3):237-45. doi: 10.1007/bfo0708730.

41. Sueishi Y, Fujita T, Nakatani S, Inazumi N, Osawa Y. The enhancement of fluorescence quantum yields of anilino naphthalene sulfonic acids by inclusion of various cyclodextrins and cucurbit[7]uril. Spectrochim Acta A Mol Biomol Spectrosc. 2013;114:344-9. doi: 10.1016/j.saa.2013.05.052. [PubMed: 23786974].

42. Buvari Á, Barcza L. $\beta$-cyclodextrin complexes of different type with inorganic compounds. Inorg Chim Acta. 1979;33:L179-80. doi: 10.1016/s0020-1693(00)89441-4.

43. Buvári-Barcza A, Rak E, Mészáros A. Inclusion complex formation of p-nitrophenol and p-nitrophenolate with hydroxypropyl- $\beta$ cyclodextrins. J Incl Phenom Mol Recognit Chem. 1998;32(4):453-9. doi: 10.1023/a:1007989822622.
44. Horsky J, Pitha J. Hydroxypropyl cyclodextrins: Potential synergism with carcinogens. J Pharm Sci. 1996;85(1):96-100. doi: 10.1021/js9501324. [PubMed: 8926593].

45. Van Cauteren H, Jacqmin P, Coussement W, Meuldermans W, Lampo A, Lammens L. Commentary on "Hydroxypropyl cyclodextrins: Potential synergism with carcinogens". J Pharm Sci. 1997;86(3):402-4. doi: 10.1021/js960299c. [PubMed: 9050815].

46. van der Veen BA, Uitdehaag JC, Penninga D, van Alebeek GJ, Smith LM, Dijkstra BW, et al. Rational design of cyclodextrin glycosyltransferase from Bacillus circulans strain 251 to increase alpha-cyclodextrin production.J Mol Biol.2000;296(4):1027-38. doi:10.1006/jmbi.2000.3528. [PubMed: 10686101]

47. Jin ZY. Cyclodextrin chemistry: Preparation and application. World Sci. 2013. doi: 10.1142/8630.

48. Szejtli J, Gerloczy A, Fonagy A. Intestinal absorption of carbon-14labeled $\beta$ - cyclodextrin in rats. Arzneim Forsch. 1980;30:808-10.

49. Szejtli J. Cyclodextrins and their inclusion complexes. Akademiai Kiado.1982;1982.

50. French D. The schardinger dextrins. Adv Carbohydr Chem. 1957;12:189260. doi: 10.1016/s0096-5332(08)60209-x.

51. Heidel JD, Schluep T. Cyclodextrin-containing polymers: versatile platforms of drug delivery materials. J Drug Deliv. 2012;2012:262731. doi: 10.1155/2012/262731. [PubMed: 22496980]. [PubMed Central: PMC3307009].

52. Jicsinszky L, Fenyvesi E, Hashimoto H, Ueno A. Cyclodextrin derivatives. In: Szejtli L, Osa T, editors. Comprehensive supramolecular chemistry. 3. USA: Elsevier; 1996. doi: 10.13140/rg.2.2.35859.68642.

53. Sugiura I, Komiyama $\mathrm{M}$, Toshima $\mathrm{N}$, Hirai $\mathrm{H}$. Immobilized $\beta$ cyclodextrins. Preparation with various crosslinking reagents and the guest binding properties. Bull Chem Soci Japan.1989;62(5):1643-51. doi: $10.1246 /$ bcsj.62.1643.

54. Tejashri G, Amrita B, Darshana J. Cyclodextrin based nanosponges for pharmaceutical use: A review. Acta Pharm. 2013;63(3):335-58. doi: 10.2478/acph-2013-0021. [PubMed: 24152895].

55. Jain A, Prajapati SK, Kumari A, Mody N, Bajpai M. Engineered nanosponges as versatile biodegradable carriers: An insight. J Drug Delivery Sci Technol. 2020;57:101643. doi:10.1016/j.jddst.2020.101643.

56. Ogino H. Relatively high-yield syntheses of rotaxanes. Syntheses and properties of compounds consisting of cyclodextrins threaded by .alpha.,.omega.-diaminoalkanes coordinated to cobalt(III) complexes. J Am Chem Soc. 1981;103(5):1303-4. doi: 10.1021/ja00395a091.

57. Komiyama M, Takeuchi T, Mukawa T, Asanuma H. Molecularimprinting from fundamentals to applications. USA: WILEY-VCH Verlag GmbH \& Co; 2002. doi: $10.1002 / 352760202 x$.

58. Lay S, Ni X, Yu H, Shen S. State-of-the-art applications of cyclodextrins as functional monomers in molecular imprinting techniques: a review. J Sep Sci. 2016;39(12):2321-31. doi: 10.1002/jssc.201600003. [PubMed: 27324352].

59. Deshmukh K, Shende P. Toluene diisocyanate cross-linked $\beta$ cyclodextrin nanosponges as a $\mathrm{pH}$-sensitive carrier for naproxen. Mater Res Express. 2018;5(7):75008. doi: 10.1088/2053-1591/aac93d.

60. Swaminathan S, Cavalli R, Trotta F, Ferruti P, Ranucci E, Gerges I, et al. In vitro release modulation and conformational stabilization of a model protein using swellable polyamidoamine nanosponges of $\beta$ cyclodextrin. J Incl Phenom Macrocyclic Chem. 2010;68(1-2):183-91. doi: 10.1007/s10847-010-9765-9.

61. Argenziano M, Haimhoffer A, Bastiancich C, Jicsinszky L, Caldera F, Trotta F, et al. In vitro enhanced skin permeation and retention of imiquimod loaded in beta-cyclodextrin nanosponge hydrogel. Pharmaceutics. 2019;11(3). doi: 10.3390/pharmaceutics11030138. [PubMed: 30897794]. [PubMed Central: PMC6471382].

62. Young Lee M, Subban Ganapathy H, Taek Lim K. Controlled drug 
release applications of the inclusion complex of peracetylated$\beta$-cyclodextrin and water-soluble drugs formed in supercritical carbon dioxide. J Phys Chem Solids. 2010;71(4):630-3. doi: 10.1016/j.jpcs.2009.12.054.

63. Horiuchi Y, Hirayama F, Uekama K. Slow-release characteristics of diltiazem from ethylated beta-cyclodextrin complexes. J Pharm Sci. 1990;79(2):128-32. doi: 10.1002/jps.2600790211. [PubMed: 2324960].

64. Umemura M, Ueda H, Tomono K, Nagai T. Effect of diethyl-betacyclodextrin on the release of nitroglycerin from formulations. Drug Des Deliv. 1990;6(4):297-310. [PubMed: 2128021].

65. Hirayama F. Inclusion ability of hydrophobic cyclodextrin derivatives and their application as controlled-release drug carrier. Adv Pharm Sci. 1990:90-8.

66. Fenyvesi É, Décsei L, Ujházy A, Zsadon B, Szejtli J. Complexes of insoluble cyclodextrin polymers. 4th Int Symp Cyclodextrins. Munich, West German. 1988. p. 227-35.
67. Crini G, Bertini S, Torri G, Naggi A, Sforzini D, Vecchi C, et al. Sorption of aromatic compounds in water using insoluble cyclodextrin polymers.J Appl Polymer Sci. 1998;68(12):1973-8. doi: 10.1002/(sici)10974628(19980620)68:12<1973::aid-app11>3.0.co;2-t.

68. Komiyama M, Hirai H. Preparation of immobilized $\beta$-cyclodextrins by use of alkanediol diglycidyl ethers as crosslinking agents and their guest binding abilities. Polymer J. 1987;19(6):773-5. doi: 10.1295/polymj.19.773.

69. Jicsinszky L, Calsolaro F, Martina K, Bucciol F, Manzoli M, Cravotto G. Reaction of oxiranes with cyclodextrins under high-energy ball-milling conditions. Beilstein J Org Chem. 2019;15:1448-59. doi: 10.3762/bjoc.15.145. [PubMed: 31354861]. [PubMed Central: PMC6632222].

70. Bednarz S, Lukasiewicz M, Mazela W, Pajda M, Kasprzyk W. Chemical structure of $\operatorname{poly}(\beta$-cyclodextrin-co-citric acid).J Appl Polymer Sci. 2011;119(6):3511-20. doi: 10.1002/app.33002. 